\title{
Validation of an Analytical Methodology for Determination of Oxytetracycline and Tetracycline Residues in Honey by HPLC with Fluorescence Detection
}

\author{
A. Pena $,{ }^{*}, \dagger$ N. Pelantova,$\stackrel{\ddagger}{ }$ C. M. Lino ${ }^{\dagger}$ M. I. N. Silveira,${ }^{\dagger}$ And P. Solich ${ }^{\ddagger}$ \\ Group of Bromatology, Centre of Pharmaceutical Studies, Faculty of Pharmacy, University of \\ Coimbra, 3000 Coimbra, Portugal, and Department of Analytical Chemistry, Faculty of Pharmacy, \\ Charles University, Prague, Czech Republic
}

\begin{abstract}
An analytical method for the determination of OTC and TC residues in honey was developed. Sample treatment involves an extraction in EDTA-Mcllvaine buffer, followed by a solid-phase cleanup step. With regard to the cleanup procedure, different SPE cartridges were evaluated and the results presented. The method was validated according to the guidelines laid down by the 2002/657/EC European Decision parameters: decision limit $(\mathrm{C} c \alpha)$ and detection capability $(\mathrm{CC} \beta)$ were 20 and 21 $\mu \mathrm{g} / \mathrm{Kg}$ and 49 and $50 \mu \mathrm{g} / \mathrm{Kg}$ for OTC and TC, respectively, and recoveries of OTC and TC from spiked samples, at three fortification levels, were higher than $87 \%$ for both compounds. The analytical method was applied to 57 honey samples.
\end{abstract}

KEYWORDS: Antibiotics; Tetracyclines; Honey; Solid-phase extraction; High-performance liquid chromatography

\section{INTRODUCTION}

In apiculture, antibiotics are mainly used for the treatment of bacterial brood diseases, American and European foulbrood (AFB and EFB) in honeybees (Apis melifera), which are caused by two species of bacteria, Paenibacillus (Bacillus) larvae and Melissococcus pluton, respectively (1).

Oxytetracycline (OTC) has been used in the United States of America since the early 1950s for the prevention and control of these diseases. Because of the easy availability and relatively low cost of OTC, this method has been widely adopted by beekeepers (2).

The occurrence of antibiotic residues in human food, arising from its veterinary use, is a cause of concern to consumers worldwide, because of possible toxic or allergic reactions and the possibility that pathogenic organisms could become resistant to these drugs $(3-4)$.

Under European Community (EC) legislation, antibiotics are not authorized for the use on honeybees, since no maximum residue levels (MRLs) have been fixed for antibiotic residues in honey in the EU Council Regulation (5).

In recent years, several initiatives have been launched to establish or strengthen surveillance systems, both in the EC member states and at the international level, to control the presence of antibiotic residues in honeys, and these have revealed levels of antibiotic residues in honey samples.

* To whom correspondence should be addressed. Tel: 00351239859994 Fax: 00351239827126. E-mail: apena@ci.uc.pt

$\dagger$ University of Coimbra.

$\doteqdot$ Charles University.
Tetracyclines (TCs) can be successfully determined in various biological matrices, using high-performance liquid chromatography (HPLC) in the reverse-phase mode, with different detection modes, such as spectrophotometry, fluorescence, and mass spectrometry (6). The UV detection has low sensitivity, while mass spectrometry still requires costly instruments. In general, fluorescence detection is sensitive and selective (7).

The present study describes the development and validation of an analytical methodology for the specific and sensitive determination of OTC and tetracycline (TC) in honey by HPLC with fluorescence detection. They were extracted with $\mathrm{Na}_{2}$ EDTA-McIlvaine buffer ( $\mathrm{pH} 4.0$ ), and different solid-phase extraction (SPE) cartridges were evaluated in order to find the most efficient cleanup method for TCs in honey samples.

To enhance the precision and accuracy of the analytical method, the validation was compliant with European Community (EC) Decision 2002/657/EC (8). Finally, the method was applied to the analysis of OTC and TC residues in different types of honeys from Portugal and Spain.

\section{EXPERIMENTAL PROCEDURES}

2.1. Apparatus. The HPLC system consisted of Gilson Model 302 and 307 pumps (Gilson, Medical Electronics, Villiers-le-Bel, France) and a Model 7125 loop injector (Rheodyne, Cotati, CA). The fluorescence detector was a Model LC 305 instrument (LabAlliance, LabAlliance, State College, PA) operating at an excitation wavelength of $385 \mathrm{~nm}$ and an emission wavelength of $500 \mathrm{~nm}$. The spectral bandwidth was $10 \mathrm{~nm}$ for both excitation and emission. The results were recorded on a Model 3390 A integrator (Hewlett-Packard, Philadelphia, PA) and a Unipoint Gilson data system (Gilson, Medical 
Electronics, Villiers-le-Bel, France). The HPLC column used was a Nucleosil $\mathrm{C}_{18}$ column $(5 \mu \mathrm{m}, 250 / 4 \mathrm{~mm})$ (Duren, Germany). The mixing $\mathrm{T}$ was directly coupled through a $60 \mathrm{~cm}(0.25 \mathrm{~mm}$ i.d. $)$ tube to the detector. The reaction temperature was about $22{ }^{\circ} \mathrm{C}$.

The mobile-phase flow was $0.8 \mathrm{~mL} \mathrm{~min}{ }^{-1}$, and the derivatization reagent was delivered at a flow rate of $0.5 \mathrm{~mL} \mathrm{~min}{ }^{-1}$.

Flatbed shaker: Edmund Buhler KL 27400 (Tubingen, Germany).

Vortex mixer: Unimag ZX (Munchen, Germany).

2.2. Reagents and Materials. Caution! Handle tetracycline standards with care, since they are irritants. Avoid contact with skin. Prepare mobile phases and all reagents in a well-ventilated chemical fume hood. HPLC grade acetonitrile and methanol were purchased from Carlo Erba (Milan, Italy). Oxalic acid, magnesium acetate, boric acid, potassium hydroxide, and sodium hydroxide (analytical reagent grade) were supplied by Merck (Darmstadt, Germany). Tetracycline and oxytetracycline standards were obtained from Sigma Chemical (Madrid, Spain).

Equipment used: Oasis HLB extraction cartridges, $3 \mathrm{~cm}^{3} / 60 \mathrm{mg}$ and $6 \mathrm{~cm}^{3} / 200 \mathrm{mg}$ (Waters Corp. Milford, MA); Bakerbond SPE carboxylic acid $(\mathrm{COOH})$ columns (J. T. Baker, Deventer, Holland); Bond Elut LRC-PRS, $500 \mathrm{mg}$ (Varian, Middelburg, The Netherlands); phenylsilane, solid-phase extraction columns, $500 \mathrm{mg}$ (J. T. Baker, Deventer, Holland); membrane filters, $0.45 \mathrm{~mm}$ HV (Millipore, Ireland).

Individual stock standard solutions were prepared in $100 \mathrm{~mL}$ of methanol containing $100 \mathrm{mg}$ of neat standard in a volumetric flask and were stored at $-20{ }^{\circ} \mathrm{C}$ in brown glass vials for a maximum period of 1 month. The working standard solutions were a mixture of the two compounds prepared by a serial dilution of the stocks in the mobile phase.

McIlvaine-EDTA buffer and reagent postcolumn were prepared as previously described (9). The mobile phase used for analysis containing aqueous oxalic acid solution $(1 \mathrm{mM})$ and $20 \%$ of acetonitrile $(\mathrm{pH} 2.0)$ was filtered through a $0.45 \mu \mathrm{m}$ filter under vacuum and degassed by ultrasonication.

Amber glassware was used to prevent photodegradation of TCs. All glassware was cleaned with Extran MA 03 (10\%; Merck, Darmstadt, Germany), washed thoroughly with tap water, rinsed with deionized water, and dried at $80{ }^{\circ} \mathrm{C}$.

2.3. Extraction and Cleanup Procedure. A sample of honey $(3.0$ g) was weighted into a polypropylene tube and dissolved in $20 \mathrm{~mL}$ of $0.1 \mathrm{M} \mathrm{Na} 2$ EDTA-McIlvaine buffer ( $\mathrm{pH} 4.0$ ). The sample solution was shaken for $5 \mathrm{~min}$ on a flatbed shaker at high speed. After filtration it was loaded on a Waters Oasis HLB $6 \mathrm{~cm}^{3}(200 \mathrm{mg})$ cartridge previously conditioned with $3 \mathrm{~mL}$ of methanol and $2 \mathrm{~mL}$ of water. The cartridge containing the sample was then washed with $5 \mathrm{~mL}$ of water, and TCs were eluted with $3 \mathrm{~mL}$ of methanol. The eluate was applied on a Varian Bond Elut LRC-PRS $(500 \mathrm{mg}$ ) cartridge previously activated with 10 $\mathrm{mL}$ of ethyl acetate. For the washing step $25 \mathrm{~mL}$ of water and $10 \mathrm{~mL}$ of methanol were used. Then the TCs were eluted with $5 \mathrm{~mL}$ of oxalic acid $(1 \mathrm{M}) /$ acetonitrile $(80 / 20)$. The final eluate was filtered through a $0.45 \mu \mathrm{m}$ membrane filter and stirred in vortex before injecting into the HPLC system. The volume of the eluate injected was $50 \mu \mathrm{L}$.

2.4 Decision Limit and Detection Capability. The $\mathrm{CC} \alpha$ value was estimated by fortifying three series of fortified blank honey at four different levels, namely $50,100,150$, and $200 \mu \mathrm{g} / \mathrm{kg}$, and the response was plotted against the added concentration. The corresponding concentration at the $y$ intercept plus 2.33 times the standard deviation (SD) of the within-laboratory reproducibility of the intercept equals the decision limit $(\mathrm{CC} \alpha)$. Furthermore, the corresponding concentration at the $\mathrm{CC} \alpha$ limit plus 1.64 times the SD of the within-laboratory reproducibility of the mean measured content at the decision limit equals the detection capability $(\mathrm{CC} \beta)(8)$.

2.5. Stability Studies. To investigate the presence of possible degradation products, a stability study of standard solutions was performed in solvent (standard solutions) and in matrix (sample purified extracts).

\section{RESULTS AND DISCUSSION}

3.1. HPLC Conditions Optimization. The methodology reported here utilizes a $\mathrm{C}_{18}$ Nucleosil column at room temper-

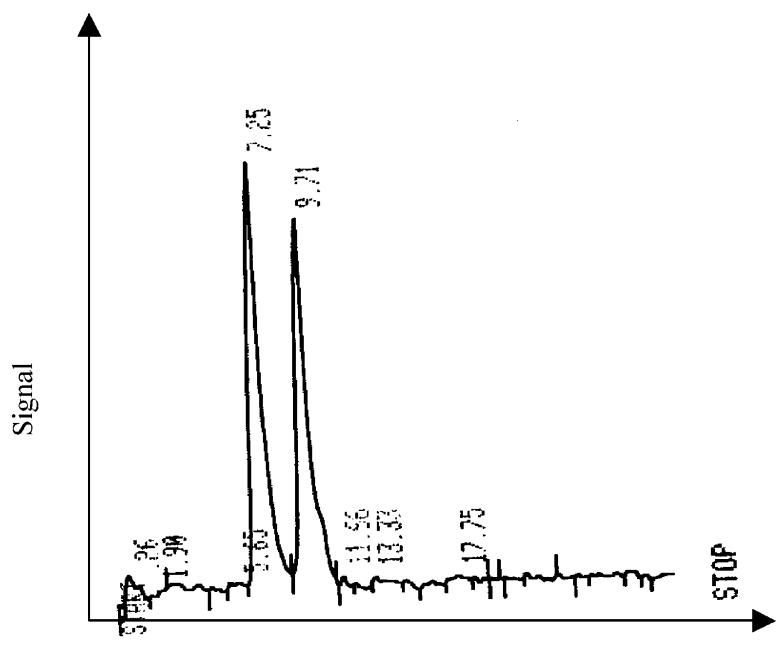

Retention time (minutes)

Figure 1. Liquid chromatogram of a standard solution of OTC and TC $(0.05 \mu \mathrm{g} / \mathrm{mL})$. Retention times of OTC and TC: 7.25 and $9.21 \mathrm{~min}$, respectively.

ature with acetonitrile/oxalate buffer $(10 \mathrm{mM})(20 / 80 ; \mathrm{pH} 2.0)$ as the mobile phase. The isocratic analysis under the conditions described allows the separation of the OTC and TC with good resolution (Figure 1). We obtain good separation and peak shape at relatively low flow rate of $0.8 \mathrm{~mL} / \mathrm{min}$.

The mean retention time for OTC and TC was 7.3 and 9.7 min, respectively. On the basis of six parallel determinations, over 5 days, the precision relative standard deviation (SD) of the retention times was $0.32 \%$ for OTC and $0.42 \%$ for TC, whereas the precision of peak area values was 1.8 and $2.1 \%$ for OTC and TC, respectively.

It must be emphasized that the analysis is performed at $\mathrm{pH}$ 2, a $\mathrm{pH}$ at which silica-based materials lack stability, and it was necessary to flush the column with a neutral solvent (e.g. water/acetonitrile, 20/80) for $1 \mathrm{~h}$ at the end of each working day. This practice contributed markedly to the prolonging of column life. The use of a guard column also provides some protection against decomposition of the column.

The present method uses the fluorescence produced when TCs react with magnesium ions, on the basis of the Haagsma and Sherpenisse method (10), optimized by Pena et al. (9). TCs are separated on a reverse-phase $\mathrm{C}_{18}$ column and reacted with magnesium acetate in $\mathrm{pH} 9.0$ boric acid buffer, at room temperature, to produce a highly fluorescent complex. This complex formation between tetracyclines and magnesium ions is used as the basis for the HPLC postcolumn reaction described here. Postcolumn derivatization has the advantage that a separate sample treatment is not required and the analytes are better separated from interferences before derivatization.

3.2. Extraction and Cleanup Optimization. The extraction with $\mathrm{pH} 4.0$ disodium ethylenediaminetetraacetate ( $\mathrm{Na}_{2}$ EDTA)McIlvaine buffer, a mild acidic solvent containing EDTA, accepted as a universal extraction method for TCs, was used.

During the method development focus was concentrated on the strong interaction of TCs with SPE cartridges, since TCs can interact by different mechanisms, including hydrophobic interactions, hydrogen bonding, chelation, and cation exchange.

The cleanup efficiencies were studied to adjust the following parameters: the type of cleanup cartridge, the solvents used in the washing steps, the eluent solvents, and the volumes for eluting TCs from the cartridges. 
When phenyl columns were used, according to the method of Vinas et al. (11), some interference could be observed at the retention times of the TCs. Under our experimental conditions, this cleanup was not efficient in removing the interferences from all different types of honeys analyzed. Afterward, we applied the cleanup described by Pagliuca et al. (12) for honey extracts through Waters Oasis HLB $3 \mathrm{~cm}^{3}$ (60 mg) cartridges. This polymeric sorbent does not contain a silanol backbone, thus avoiding the problem of TCs interacting too strongly with the silanols of a silica-based cartridge. To optimize the experimental conditions, two amounts of this sorbent, 60 and $200 \mathrm{mg}$, with the same particle size, were assayed. Because of the consistently reproducible recoveries of TCs, Oasis $200 \mathrm{mg}$ cartridges were chosen for this study, but the chromatograms also presented some interfering peaks, corresponding to substances which might have originated in a matrix effect.

It is important to note that, in both of the procedures described above, more interference in the blanks of some dark honey samples was observed. For light-colored honey and some colored honeys we obtained more clean blanks, but for other dark-colored honeys the blank presented more interferences. This can be explained by the fact that the honey samples can vary in terms of complexity/content in natural products.

As was the case for other authors $(13,14)$, our findings show that a more effective cleanup procedure that can separate TCs from impurities present in all types of honeys is essential. Oka et al. (13) also observed that a single cleanup ( $\mathrm{C}_{18}$ cartridge) was not always sufficient for the residual analysis of TCs in some samples of honey. To eliminate the interferences, and on the basis of the method of Oka et al. (13), we proceeded to a second cleanup through a $\mathrm{COOH}$ column. Solvents used for washing steps, eluent solvents, and their volumes were evaluated for $\mathrm{COOH}$ cartridges. Methanol was more efficient in removing the interferences, in comparison with water. Therefore, it was used for washing the $\mathrm{COOH}$ columns. Ethyl acetate, mobile phase, and ethyl acetate acidified at $\mathrm{pH} 2.0$ were tested for efficient elution of TCs. The overall recovery efficiency of TCs was as follows: mobile phase $>$ ethyl acetate acidified $>$ ethyl acetate. Then, the following volumes of the eluent selected were assayed: 1,2 , and $5 \mathrm{~mL}$. Ours results show that $1 \mathrm{~mL}$ is not sufficient to elute all TCs (OTC 32\%, TC 24\%), with $2 \mathrm{~mL}$ of mobile phase all TCs were eluted (OTC 99\%, TC 89\%), and no improvement was observed in a further elution with $2 \mathrm{~mL}$. Following this methodology, we obtained clean blanks, but the recovery assays performed on different days, with different samples, showed a large variation in concentrations: between 32 and $99 \%$ for OTC and 24 and $88 \%$ for TC.

To minimize the losses of the method, in order to improve the method precision, we compared the results obtained for filtering through a paper filter and glass wool and when applying the sample extracts without filtration. Neither of the filtrations result in more reproducible recoveries, and the passage of the extract directly through the column leads to clogging problems.

Our results are in accordance with a previously published paper (14), although $\mathrm{C}_{18}$ cartridges were not used for the cleanup. Geertsen and Pederson (14) applied Oka's method (13) on honey samples and obtained values for recovery lower than $40 \%$ and higher than $90 \%$ when standards without matrix were applied. The different $\mathrm{pH}$ values of the honey samples could be the reason for these observations, since retention of analytes on the $\mathrm{COOH}$ column was affected. By changing to a strong cationic exchanger (PRS) the problem with $\mathrm{pH}$ was solved, since the $\mathrm{p} K_{\mathrm{a}}$ of PRS is very low $(<1)$. For that reason PRS cartridges were selected for the second cleanup, according to the Geertsen and Pederson method (14).

To improve this second cleanup, $10 \mathrm{~mL}$ of water was also used for washing the column. The chromatograms of the blank assays show less interference, so we concluded that water was a good wash solvent. Interferences decrease with an increase of the volume of water used. Consequently, an optimized 25 $\mathrm{mL}$ of water was used as the final volume. The best blank assays were obtained when $25 \mathrm{~mL}$ of water and $10 \mathrm{~mL}$ of methanol were applied on the PRS column to remove the interferences.

Several solvents were tested for efficient elution of TCs: oxalic acid $(10 \mathrm{mM}) /$ acetonitrile (80/20, $\mathrm{pH} 2.0)$; oxalic acid (10 mM)/acetonitrile (70/30, $\mathrm{pH} 2.0)$; oxalic acid $(10 \mathrm{mM}) /$ acetonitrile/methanol (70/15/15, pH 2.0), and oxalic acid (1 M)/ acetonitrile (80/20, $\mathrm{pH} 1.4)$. Our results show that $5 \mathrm{~mL}$ of oxalic acid (1 M)/acetonitrile (80/20, $\mathrm{pH} 1.4)$ is necessary to elute all TCs from the PRS column with high recoveries: OTC, $86 \%$; TC, $93 \%$.

To verify the absence of interfering substances around the retention times of OTC and TC, 15 colored honey samples were analyzed. No interference was detected in any of the honeys analyzed. Thus, this method was applied to analyze TC residues in honey samples.

3.3 Analytical Method Validation. To verify the absence of potential interfering compounds around the retention time of OTC and TC, a number of representative blank honey samples from different origins $(n=15)$ were analyzed in order to assess the specificity of the method. No interferences were observed in the region of interest where the analytes were eluted.

The calibration curves were obtained using the linear leastsquares regression procedure of the peak area versus the concentration. The linearity for OTC and TC, in the working standard solution concentration range between 0.025 and 1.0 $\mu \mathrm{g} / \mathrm{mL}$, was good, as shown by the fact that the determination of the correlation coefficients $\left(r^{2}\right)$ is above 0.9990 for 10 calibration curves, prepared on three different days.

In compliance with the Decision 2002/657/EC (8) concerning the performance of methods, $\mathrm{CC} \alpha$ and $\mathrm{CC} \beta$ were elaborated using three different assays of blank honey samples fortified at $50,100,150$, and $200 \mu \mathrm{g} / \mathrm{Kg}$. The matrix calibration curves were prepared by spiking blank honey samples with a standard solution of OTC and TC, and the correlation coefficients were greater than 0.991 for all curves.

The CC $\alpha$ values obtained were 20 and $21 \mu \mathrm{g} / \mathrm{Kg}$, for OTC and TC, respectively. The concentration at the decision limit plus 1.64 times the standard deviation of the within-laboratory reproducibility of the mean measured content at the decision limit equals the detection capability $(\mathrm{CC} \beta)$. The $\operatorname{CC} \beta$ values obtained for OTC and TC were 49 and $50 \mu \mathrm{g} / \mathrm{Kg}$, respectively.

The recovery and repeatability of the method were evaluated by the analysis of six spiked samples with OTC and TC at 50, 100 , and $200 \mu \mathrm{g} / \mathrm{Kg}$, on three different days $(n=18)$. Under our conditions, the estimated extraction recoveries from spiked honey samples for OTC and TC reached very high values, ranging from 86 to $95 \%$ with low relative standard deviation (RSD) (Table 1). For example, the recoveries for OTC and TC at $100 \mu \mathrm{g} / \mathrm{Kg}$ were $91 \pm 4 \%$ and $90 \pm 5 \%$, respectively. Figure 2 represents the chromatogram obtained for fortification assay at $50 \mu \mathrm{g} / \mathrm{Kg}$.

Inter- and intra-day repeatability has been established for six measurements of spiked honey samples with OTC and TC at three concentrations, carried out within the same day and on three different days. The percent relative standard deviation for 
Table 1. Accuracy and Inter- and Intraassay Validation Results

\begin{tabular}{cccccc}
\hline & $\begin{array}{c}\text { no. of } \\
\text { assays } \\
\text { tetracycline }\end{array}$ & $\begin{array}{c}\text { cortification } \\
\text { level }(\mu \mathrm{g} / \mathrm{kg})\end{array}$ & $\begin{array}{c}\text { recovery } \\
\text { mean }(\%)\end{array}$ & $\begin{array}{c}\text { within- } \\
\text { day }\end{array}$ & $\begin{array}{c}\text { between- } \\
\text { day }\end{array}$ \\
\hline OTC & 5 & 50 & 86 & 6 & 8 \\
TC & 5 & 50 & 93 & 6 & 8 \\
OTC & 5 & 100 & 91 & 4 & 7 \\
TC & 5 & 100 & 90 & 5 & 6 \\
OTC & 5 & 200 & 93 & 4 & 7 \\
TC & 5 & 200 & 95 & 5 & 6 \\
\hline
\end{tabular}

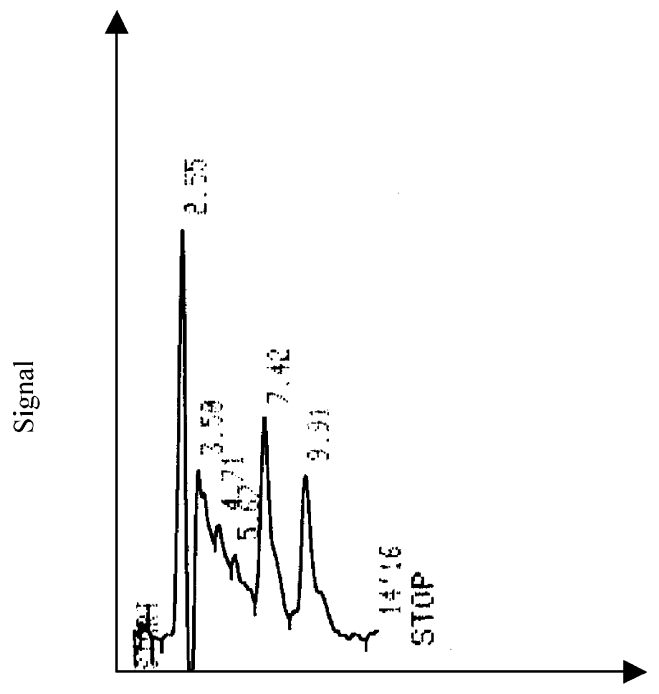

Retention time (minutes)

Figure 2. Liquid chromatogram of a fortified assay at $50 \mu \mathrm{g} / \mathrm{kg}$. Retention times of OTC and TC: 7.42 and 9.91 min, respectively.

all fortification levels was less than 11, for intra-day repeatability, demonstrating the good method precision.

3.4. Stability. The stock standard solutions, prepared in methanol, were stored at $-20{ }^{\circ} \mathrm{C}$ and analyzed over a 1 month period. For the period of study we did not observed any degradation of TCs.

The working standard solutions, prepared in the mobile phase at $\mathrm{pH} 2.0$, were stored at $0-4{ }^{\circ} \mathrm{C}$ and analyzed over a 1 week period, since the stability of these antibiotics is poor under strong acidic conditions, as epimerization on carbon- 4 in TCs can occur. Furthermore, the presence of a hydroxyl group at $\mathrm{C}_{6}$ favors dehydration and aromatization of the $\mathrm{C}$ ring of TCs following pseudo-first-order kinetics, leading to anhydrotetracyclines (ATCs) at very low $\mathrm{pH}$. The epimerization of the ATCs and the dehydration of the epitetracyclines lead to the formation of epianhydrotetracyclines (15).

Although under our chromatographic conditions we could not achieve the separation of OTC and TC from its epimers, we have observed an alteration of the peak shape of TC and also the presence of a peak eluted after TC, in the working standard solutions, on the sixth day. To check the stability of purified extracts of spiked samples at $50 \mu \mathrm{g} / \mathrm{Kg}$, stored at $4{ }^{\circ} \mathrm{C}$, they were reanalyzed also over a 1 week period, and similar results were observed.

3.5. Application to Real Samples. A total of 57 honey samples were purchased in different local markets and from several beekeepers, 26 from Valencian Community in Spain, in November 2002, 29 from the central zone of Portugal collected during the years 2001 and 2003, and two commercial samples with label indication of imported honeys, available in the Portuguese market in 2003. Both Portuguese and Spanish honey samples were provided to the markets from the beekeeper associations of these respective areas, ensuring that they were provided in the zone under study.

All Portuguese samples were of rosemary and multiflower origin, and the Spanish samples were from different floral origins: thyme, multiflowers, rosemary, heather, lavender, orange blossom, lemon, acorn, and eucalyptus. Four of them were ecological honeys. All samples were stored in glass jars in a dark and dry place at room temperature.

None of the honey samples analyzed showed contamination of OTC and TC residues at detectable levels.

\section{CONCLUSIONS}

An HPLC method with fluorescence detection for the determination of OTC and TC residues in honey was developed and validated according to the Decision 2002/657/EC, and the results obtained fulfill the requirements defined.

The cleanup procedure is a critical step, as honey is a complex matrix. Double cleanup using a combination of two SPE cartridges is necessary in order to eliminate interferences, and the best recovery results were obtained for polymeric and ionexchange cartridges.

A total of 57 samples of Spanish, Portuguese, and imported honeys of different botanical origin were examined. None of the honey samples analyzed showed contamination of OTC and $\mathrm{TC}$ residues at detectable levels.

\section{LITERATURE CITED}

(1) Kochanski, J. Analysis of oxytetracyclinein extender patties. Apidologie 2000, 31, 517-524.

(2) Sporns, P.; Khan, S; Roth, L. A. HPLC analysis of oxytetracycline residues in honey. J. Food Protect. 1986, 49, 383-388.

(3) van den Bogaard, A. E.; Stoobberingh, E. E. Epidemiology of resistance to antibiotics. Links beteween animals and humans. Int. J. Antimicrob. Agents 2000, 14, 327-335.

(4) Sáenz, Z.; Zarazaga, M.; Brinas, L., Lantero, M.; Ruiz-Larrea, F.; Torres, C.; Antibiotic resistance in Escherichia coli isolates obtained from animals, foods and humans in Spain. Int. J. Antimicrob. Agents 2001, 18, 353-358.

(5) Establishment of Maximum Residue Levels of Veterinary Medical Products in foodstuffs of animal origin, Council Regulation (EEC) No. 2377/90, Off. J. Eur. Commun. L224/1, 1990.

(6) Oka, H.; Patterson, J. Chemical analysis of tetracyclines. In Chemical Analysis for Antibiotics Used in Agriculture; Oka, H., Nakazawa, N., Harada, K., MacNeil, J. D., Eds.; AOAC: Gaithersburg, MD, 1995; pp 333-405.

(7) Iwaki, K.; Okumura, N.; Yamazaki, M. Determination of tetracycline antibiotics by reversed phase high-performance liquid chromatography with fluorescence detection. J. Chromatogr. 1992, 623, 153-158.

(8) Implementing Council Directive 96/23/EC concerning the performance of analytical methods and the interpretation of results, EC Decision 2002/657, Off. J. Eur. Commun. L221/8, 2002.

(9) Pena, A. L. S.; Lino, C. M.; Silveira, M. I. N. Determination of tetracycline antibiotics in salmon muscle by liquid chromatography using postcolumn derivatization with fluorescence detection. J. Assoc. Off. Anal. Chem. Int. 1999, 82, 55-60.

(10) Haagsma, N.; Scherpenisse, P. In Proceedings of Euroresidue II; Haagsma, N., Ruiter, A., Czedik-Eysenberg, J., Eds.; Euroresidue II Conference; Veidhoven, The Netherlands, 1993; p 342

(11) Vinas, P.; Balsalobre, N.; Hernández-Córdoba, L.-E. Liquid determination with ultraviolet absosbance detection for the analysis of tetracycline residues in honey. J. Chromatogr. A 2004, $1022125-129$ 
(12) Pagliuca, G.; Gazotti, T., Serra, G., Sabatini, A. G. A scientific note on the determination of oxytetracycline residues in honey by high-performance liquid chromatography with UV detection. Apidologie 2002, 33, 583-584.

(13) Oka, H.; Ikai, Y.; Kawamura, N.; Uno, K.; K; Harada, K. I.; Suzuki, M. Improvement of chemical analysis of antibiotics XII: Simultaneous analysis of seven tetracyclines in honey. $J$. Chromatogr. 1987, 389, 417-426.

(14) Geertsen, G.; Pederson, B. Determination of residual tetracyclines in honey by HPLCUV: Optimization and validation. In Proceedings of Euroresidue IV Conference. Residues of Veterinary Drugs in Foods; van Ginkel, L. A., Ruiter, A., Eds.; Veidhoven, The Netherlands, 2000; pp 460-464.
(15) Khan, N. H.; Wera, P.; Roets, E.; Hoogmartens, J. Quantitative analysis of tetracycline by high-performance liquid chromatography on polystyrenedivinylbenzene packing materials. J. Liq. Chromatogr. 1990, 13, 1351-1374.

Received for review January 12, 2005. Revised manuscript received March 18, 2005. Accepted March 18, 2005. We thank the FCT/FSE (Fundação para a Ciência e Tecnologia/Fundo Social Europeu) for financial support of this project.

JF050065R 